\title{
Deformed SW curve and the null vector decoupling equation in Toda field theory
}

\author{
Rubik Poghossian \\ Yerevan Physics Institute, \\ Alikhanian Br. 2, AM-0036 Yerevan, Armenia \\ E-mail: poghos@yerphi.am
}

ABSTRACT: It is shown that the deformed Seiberg-Witten curve equation after Fourier transform is mapped into a differential equation for the AGT dual 2d CFT cnformal block containing an extra completely degenerate field. We carefully match parameters in two sides of duality thus providing not only a simple independent prove of the AGT correspondence in Nekrasov-Shatashvili limit, but also an extension of AGT to the case when a secondary field is included in the CFT conformal block. Implications of our results in the study of monodromy problems for a large class of $n$ 'th order Fuchsian differential equations are discussed.

KeYwords: Conformal and W Symmetry, Nonperturbative Effects, Supersymmetric gauge theory, Integrable Field Theories

ARXIV EPRINT: 1601.05096 


\section{Contents}

1 Introduction 1

2 Deformed Seiberg-Witten curve for $A_{r}$ quiver 3

2.1 Exponents 7

2.1.1 Points $z_{r+2}=0$ and $z_{0}=\infty \quad 7$

2.1.2 Points $z_{1}=1, z_{2}=q_{1}, z_{3}=q_{1} q_{2}, \ldots, z_{r+1}=q_{1} q_{2} \cdots q_{r} \quad 7$

3 Toda CFT $\quad 8$

3.1 Preliminaries on $A_{n-1}$ Toda CFT 8

3.2 Fusion with the completely degenerated field $V_{-b \omega_{1}} \quad 9$

3.3 Derivation of null-vector decoupling equation in semiclassical limit $\quad 10$

4 Comparison with the differential equation derived from DSW 15

4.1 From the gauge theory differential equation to the null-vector decoupling equation

4.2 Matching $S_{2}(z)$ with $w^{(2)}(z)$ : emergence of AGT

$4.3 A_{n-1}$-Toda 4-point functions versus $\mathrm{SU}(n)$ gauge theory with $2 n$ fundamental hypers

\section{Introduction}

Low energy behavior of $N=2$ SYM theory admits an exact description including both perturbative and non-perturbative layers [1, 2]. All relevant quantities, such as the prepotential and chiral gauge invariant expectation values are nicely encoded in the geometry of Riemann surfaces, called in this context the Seiberg-Witten curve. It was realized from the very beginning that this curve is intimately related to the classical integrable systems $[3,4]$. Later development of this field was triggered by the application of localization method [5-8]. An earlier important reference is [9]. To make localization method efficient one should first formulate the theory in a non-trivial background, commonly referred as the $\Omega$-background [5, 8], which is parameterized by two numbers $\epsilon_{1}, \epsilon_{2}$ (these are rotation angles in $\left(x^{1}, x^{2}\right)$ and $x^{3}, x^{4}$ planes of Euclidean space time). The $\Omega$-background brakes the Poincaré symmetry and effectively regularizes the space-time volume, making the partition function finite. Using localization the instanton part of the partition function as well as the chiral correlators of the theory can be represented as sum over arrays of Young diagrams in such a way, that their total number of boxes coincides with the instanton number. Sending the parameters $\epsilon_{1,2}$ to zero one recovers the known results of the trivial background. It appears, nevertheless, that the theory on finite $\Omega$-background has its own significance. Namely, the recent developments recovered intriguing relations of these theory 
with 2d CFT called the AGT correspondence [10-12]. According to AGT correspondence the partition functions of gauge theories get identified with 2d CFT conformal blocks.

An interesting special case of the $\Omega$-background is the Nekrasov- Shatashvili limit [13] when only one of the parameters, say $\epsilon_{1} \rightarrow 0$. In this limit the classical integrable system associated with SW curve gets quantized so that the remaining parameter $\epsilon_{2}$ plays the role of the Plank's constant. In [14-17] this limit has been investigated using Bohr-Sommerfeld semiclassical method. Another approach initiated in [18] and further developed in [19-21] is based on the careful analysis of the contributions of various arrays of Young diagrams. It was shown in [18] that there is a single array of diagrams which dominates in the NS limit. This approach leads to a generalization of the notion of Seiberg-Witten curve. The algebraic equations defining SW curve get replaced by difference equations (referred as deformed Seiberg-Witten curve or shortly DSW equations). It is worth noting that like the original SW curve, DSW "curve" besides the prepotential encodes information about all chiral correlators. Let me describe briefly how DSW equation emerges. One starts with an entire function whose zeros encode the lengths of the rows of the dominant array of Young diagrams mentioned above. The condition of giving the most important contribution is translated then to a linear difference equation for this entire function [18]. The DSW equation (no longer linear) emerges as a condition on the ratio of this entire function with itself with a shifted argument. The initial linear difference equation is closely related to the Baxter's $T-Q$ equation which plays an important role in the context of exactly integrable statistical mechanics [22] and QFT models [23]. Fourier transform of the linear difference leads to a linear differential equation. This is the same equation which emerged as the Schrödinger equation in the already mentioned alternative Bohr-Sommerfeld approach to the NS limit.

For the purposes of this paper it is essential that from the AGT perpective the NS limit corresponds to the classical $(c \rightarrow \infty)$ limit of 2 d CFT conformal block of "heavy" fields. The idea to apply DSW equation to investigate semiclassical limit of $2 \mathrm{~d}$ CFT was suggested in [18]. For the alternative approaches to the NS limit and the semiclassical CFT see e.g. [16, 17, 24-26]. The case of irregular conformal blocks is considered in [27]. From the AGT point of view the linear differential equation discussed in previous paragraph appears to be closely related to the null-vector decoupling equation in $2 \mathrm{~d}$ CFT. Some results in this direction has been already announced in [21]. For applications of CFT degenerate fields in AGT context see also [28-32].

In this paper we systematically Investigate this relationship in a quite general setting of $A_{r}$ linear quiver theories with an arbitrary number (equal to $r$ ) of $\mathrm{SU}(n)$ gauge groups corresponding in AGT dual CFT side to the $r+3$-point conformal blocks in $W_{n}$ Toda field theory.

The subsequent material is organized as follows. In section 2 we investigate DSW equations for $A_{r}$ quiver theories and establish explicit relations between curve parameters and chiral expectation values. Then using Fourier transform we derive the corresponding linear differential equation and thoroughly investigate its singular points. In section 3 starting from the general structure of the fusion rule of the completely degenerated field $V_{-b \omega_{1}}$ and using Ward identities together with some general requirements necessary to get 


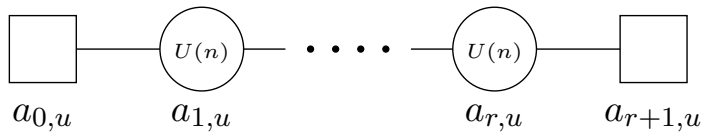

(a)

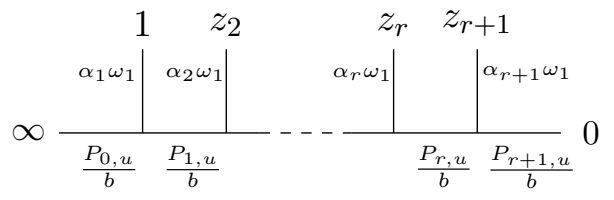

(b)

Figure 1. (a) The quiver diagram for the conformal linear quiver $\mathrm{U}(n)$ gauge theory: $r$ circles stand for gauge multiplets; two squares represent $n$ anti-fundamental (on the left edge) and $n$ fundamental (the right edge) hypermultiplets; the lines connecting adjacent circles are the bi-fundamentals. (b) The AGT dual conformal block of the Toda field theory.

acceptable solutions, we derive the null-vector decoupling equation in the semiclassical limit. Note that our approach here is somewhat heuristic and seems to be applicable only in semiclassical case. To get exact differential equation valid in full pledged quantum case one should construct the null vector explicitly and make use of the complicated $W_{n}$-algebra commutation relations and Ward identities. To my knowledge, at least in its full generality, this goal has not been achieved yet. In section 4 we show that under a simple transformation the two differential equation of previous sections can be completely matched. Already comparison of the first non-trivial coefficient functions (in $2 \mathrm{~d}$ CFT side this function is the classical expectation value of the stress-energy tensor) readily recovers the celebrated AGT correspondence. Thus our analysis provides a new, surprisingly elementary proof of the AGT duality in semiclassical limit. Matching further coefficient functions (i.e. the higher spin $W$-current expectation values in $2 \mathrm{~d}$ CFT side and their gauge theory counterparts) extends the scope of AGT correspondence: the conformal blocks including a descendant field get related to the higher power chiral expectation values in gauge theory. This new relations are explicitly demonstrated in full details in the case $r=1$ corresponding to the four-point conformal blocks. Finally in Conclusion we emphasize the relevance of our findings in the context of the monodromy problems in a large class of Fuchsian differential equations.

\section{Deformed Seiberg-Witten curve for $A_{r}$ quiver}

Partition function and chiral correlators of $\mathcal{N}=2$ gauge theory can be represented as sum over arrays of Young diagrams which label the fixed points of space time rotations and global gauge transformations acting in the moduli space of instantons. [5-8]. In the case of $A_{r}$ quiver theory with fundamental and bi-fundamental matter hypermultiplets and unitary $\mathrm{U}(n)$ gauge groups (see figure 1a), there is an $n$-tuple of Young diagrams associated to each of the $r$ gauge groups (indicated by circles in figure 1a). It has been shown in [18] for the case of a single gauge group and later generalized further in [19-21] that among all fixed points in moduli space of instantons there is a unique one giving a non-vanishing 
contribution in the Nekrasov Shatashvili limit. ${ }^{1}$ We will denote the (rescaled by $\epsilon_{1}$ ) lengths of the rows of this "critical" array of Young diagrams $Y_{\alpha, u}$ by $\lambda_{\alpha, u, i}$ where $\alpha=1, \ldots r$ refers to the node of the quiver, $u=1, \ldots, n$ is the index of the defining representation of the gauge group $\mathrm{U}(n)$ associated with this node and $i=1,2, \ldots$ specifies the row. The data $\lambda_{\alpha, u, i}$ can be conveniently encoded in meromorphic functions $y_{\alpha}(x)$, which are endowed with zeros located at $x=a_{\alpha, u}+i-1+\lambda_{\alpha, u, i}$ and poles at $x=a_{\alpha, u}+i-2+\lambda_{\alpha, u, i}$ where $a_{\alpha, u}$ are the Coulomb branch parameters. In addition we associate to the "frozen" nodes (indicated by squares in figure 1a) the parameters $a_{0, u}$ and $a_{r+1, u}$. In terms of these parameters the masses of fundamental and anti-fundamental hypermultiplets are given by

$$
m_{u}=a_{r+1, u}-\frac{1}{n} \sum_{v=1}^{n} a_{r, u} \quad \text { and } \quad \bar{m}_{u}=a_{0, u}-\frac{1}{n} \sum_{v=1}^{n} a_{1, v}
$$

respectively. In terms of

$$
\bar{a}_{\alpha}=\frac{1}{n} \sum_{u=1}^{n} a_{\alpha, u}
$$

the masses of the bifundamental hypermultiplets are simply

$$
m_{\alpha, \alpha+1}=\bar{a}_{\alpha+1}-\bar{a}_{\alpha} .
$$

The Deformed Seiberg-Witten (DSW) curve equations arise from the condition on the instanton configuration to give the most important contribution to the prepotential in NS limit. In the case of our present interest of $A_{r}$ quiver theory we get a system of $r$ (difference) equations for $r$ functions $y_{\alpha}(x), \alpha=1, \ldots, r$. In addition we introduce two polynomials

$$
y_{0}(x)=\prod_{u=1}^{n}\left(x-a_{0, u}\right) ; \quad y_{r+1}(x)=\prod_{u=1}^{n}\left(x-a_{r+1, u}\right)
$$

which encode fundamental hyper-multiplets attached to the first and the last nodes of the quiver figure 1a. The equations can be found using iterative procedure based on so called iWeyl reflections (i stands for instanton) [33]

$$
y_{\alpha}(x) \rightarrow y_{\alpha}(x)+\frac{y_{\alpha-1}(x-1) y_{\alpha+1}(x)}{y_{\alpha}(x-1)}
$$

It appears that the result of this procedure is related to the $q$-Character of the $\alpha$ 'th fundamental representation of the group $A_{r}$. Explicitly for $\alpha=1,2, \ldots, r$ one obtains ${ }^{2}$

$$
\chi_{\alpha}(x)=y_{0}(x-\alpha) \sum_{1 \leq k_{1}<k_{2}<\cdots<k_{\alpha} \leq r+1} \prod_{\beta=1}^{\alpha}\left(\frac{y_{k_{\beta}}(x-\alpha+\beta)}{y_{k_{\beta}-1}(x-\alpha+\beta-1)} q_{\beta}^{\beta-\alpha} \prod_{\gamma=1}^{k_{\beta}-1} q_{\gamma}\right),
$$

\footnotetext{
${ }^{1}$ For simplicity in this paper we'll set $\epsilon_{2}=1$. This is not a loss of generality since a generic $\epsilon_{2}$ everywhere can be restored by simple scaling arguments.

${ }^{2}$ When comparing this formula with those of [20,21] it should be taken into account that we have shifted arguments in $y_{\alpha}(x), \chi_{\alpha}(x)$ appropriately to get rid of explicit appearance of the bifundamental masses.
} 
where $q_{\alpha}$ are the gauge couplings, $\chi_{\alpha}(x)$ are $n$-th order polynomials in $x$ with coefficients related to the expectation values $\left\langle\operatorname{tr} \phi_{\alpha}^{J}\right\rangle$ ( $\phi_{\alpha}$ are the scalars of the vector multiplet) in a way to be specified below. For later purposes we'll set by definition $\chi_{0}(x) \equiv y_{0}(x)$ and $\chi_{r+1}(x) \equiv y_{r+1}(x)$. The difference equations (2.6) are the deformed Seiberg-Witten equations [18] for the $A_{r}$ quiver gauge theory [20, 21]. It is assumed that all functions $y_{\alpha}(x)$ are normalized so that their large $x$ expansions read

$$
y_{\alpha}(x)=x^{n}\left(1-c_{\alpha, 1} x^{-1}+c_{\alpha, 2} x^{-2}-c_{\alpha, 3} x^{-3}+\cdots\right) .
$$

The 1-forms $d \log y_{\alpha}(x)$ are the direct analogs of Seiberg-Witten differentials and define the chiral correlators by the conventional contour integrals

$$
\left\langle\operatorname{tr} \phi_{\alpha}^{J}\right\rangle=\oint_{\gamma_{\alpha}} \frac{d x}{2 \pi i} x^{J} \partial_{x} \log y_{\alpha}(x)
$$

where $\gamma_{\alpha}$ are large contours surrounding all zeros and poles of $y_{\alpha}(x)$ in anti-clockwise direction. Comparison of (2.7), with (2.8) allows one to express the expansion coefficients $c_{\alpha, k}$ in terms of chiral correlators $\left\langle\operatorname{tr} \phi_{\alpha}^{J}\right\rangle$ with $J \leq k$. Here are the first few relations

$$
\begin{aligned}
& \left\langle\operatorname{tr} \phi_{\alpha}\right\rangle=c_{\alpha, 1} \\
& \left\langle\operatorname{tr} \phi_{\alpha}^{2}\right\rangle=c_{\alpha, 1}^{2}-2 c_{\alpha, 2} \\
& \left\langle\operatorname{tr} \phi_{\alpha}^{3}\right\rangle=c_{\alpha, 1}^{3}-3 c_{\alpha, 1} c_{\alpha, 2}+3 c_{\alpha, 3} \\
& \left\langle\operatorname{tr} \phi_{\alpha}^{4}\right\rangle=c_{\alpha, 1}^{4}-4 c_{\alpha, 1}^{2} c_{\alpha, 2}+4 c_{\alpha, 1} c_{\alpha, 3}+2 c_{\alpha, 2}^{2}-4 c_{\alpha, 4}
\end{aligned}
$$

On the other hand, inserting the expansion (2.7) into (2.6) and comparing left and right hand sides one can express the coefficients $c_{\alpha, k}$ (and due to (2.9) also $\left\langle\operatorname{tr} \phi_{\alpha}^{J}\right\rangle$ ) in terms of coefficients of the polynomials $\chi_{\alpha}(x)$. In fact the first $n$ of these relations can be inverted to get the coefficients of the polynomials $\chi_{\alpha}(x)$ in terms of $c_{\alpha, 1}, \ldots, c_{\alpha, n}$ (or, equivalently, in terms of $\left.\left\langle\operatorname{tr} \phi_{\alpha}\right\rangle, \ldots,\left\langle\operatorname{tr} \phi_{\alpha}^{n}\right\rangle\right)$. Then the remaining infinite number of relations are nothing but the deformation of the celebrated chiral ring relations [34] expressing higher power $(J>n)$ chiral expectation values $\left\langle\operatorname{tr} \phi_{\alpha}^{J}\right\rangle$ in terms of lower, up to the $n$ 'th power expectation values. $^{3}$ For our later purposes let us display explicitly the relations for the first three coefficients of the polynomials

$$
\chi_{\alpha}(x)=\sum_{i=0}^{n}(-)^{i} \chi_{\alpha, i} x^{n-i}
$$

\footnotetext{
${ }^{3}$ For the generalization of chiral ring relation for the generic $\Omega$-background see [35].
} 
Expanding 1.h.s. of (2.6) up to the order $\sim x^{n-3}$ we get

$$
\begin{aligned}
\chi_{\alpha, 0}= & \sum_{1 \leq k_{1}<\cdots<k_{\alpha} \leq r+1} \prod_{\beta=1}^{\alpha} \prod_{\gamma=\beta}^{k_{\beta}-1} q_{\gamma} ; \\
\chi_{\alpha, 1}= & \sum_{1 \leq k_{1}<\cdots<k_{\alpha} \leq r+1}\left(c_{0,1}-\sum_{\beta=1}^{\alpha}\left(c_{k_{\beta}-1,1}-c_{k_{\beta}, 1}\right)\right) \prod_{\beta=1}^{\alpha} \prod_{\gamma=\beta}^{k_{\beta}-1} q_{\gamma} ; \\
\chi_{\alpha, 2}= & \sum_{1 \leq k_{1}<\cdots<k_{\alpha} \leq r+1}\left[\sum_{\beta=1}^{\alpha-1} \sum_{\gamma=\beta+1}^{\alpha}\left(c_{k_{\beta}-1,1}-c_{k_{\beta}, 1}+2\right)\left(c_{k_{\gamma}-1,1}-c_{k_{\gamma}, 1}+2\right)\right. \\
& +\sum_{\beta=1}^{\alpha}\left(c_{k_{\beta}-1,1}\left(-c_{k_{\beta}, 1}+\alpha-\beta+3\right)-\left(c_{0,1}+2 \alpha\right)\left(c_{k_{\beta}-1,1}-c_{k_{\beta}, 1}+2\right)\right. \\
& \left.+(-\alpha+\beta-2) c_{k_{\beta}, 1}+c_{k_{\beta}-1,1}^{2}-c_{k_{\beta}-1,2}+c_{k_{\beta}, 2}+2 \alpha-2 \beta+3\right) \\
& \left.+\alpha\left(c_{0,1}+\alpha\right)+c_{0,2}\right] \prod_{\beta=1}^{\alpha} \prod_{\gamma=\beta}^{k_{\beta}-1} q_{\gamma} .
\end{aligned}
$$

Clearly with more efforts it should be possible to write down expressions for further coefficients but unfortunately these expressions soon become quite intractable. In section 4 we'll do one more step giving an explicit expression for the next coefficient in the special case when $r=1$.

Quite remarkably it is possible to eliminate the functions $y_{2}(x), \ldots, y_{r}(x)$ from eq. (2.6) and find a single equation for $y_{1}(x)$. Here is the result: ${ }^{4}$

$$
1+\sum_{i=1}^{r+1}(-)^{i} \frac{\chi_{i}(x)}{y_{1}(x)} \prod_{j=1}^{i-1} \frac{y_{0}(x-j)}{y_{1}(x-j)} q_{j}^{i-j}=0,
$$

It is useful to represent the meromorphic functions $y_{1}(x)$ as a ratio:

$$
y_{1}(x)=y_{0}(x) \frac{Y(x)}{Y(x-1)},
$$

where $Y(x)$ is an entire function with zeros located at $x=a_{1, u}+(i-1)+\lambda_{1, u, i}$ (remind that $\lambda_{\alpha, u, i}$ is the appropriately rescaled length of the $i$ 'th row of the Young diagram $Y_{\alpha, u}$ ). In terms of $Y(x)$ the eq. (2.14) can be rewritten as

$$
\sum_{\alpha=0}^{r+1}(-)^{\alpha}\left(\prod_{\beta=1}^{\alpha} q_{\beta}^{\alpha-\beta}\right) \chi_{\alpha}(x) Y(x-\alpha)=0 .
$$

Since for small values of the gauge couplings $q_{\alpha} \ll 1$ the $i$ 'th row length $\lambda_{\alpha, u, i} \rightarrow 0$ when $i \rightarrow \infty$, it is reasonable to expect that the sum

$$
\psi(z)=\sum_{x \in \mathcal{Z}+a_{1, u}} Y(x) z^{-x}
$$

\footnotetext{
${ }^{4}$ Of course, the same can be done also for $y_{r}(x)$.
} 
will converge in some ring with the center located at 0 . Then the difference equation for $Y(x)$ can be easily "translated" into a linear differential equation for $\psi(x)[19,20]$

$$
\sum_{\alpha=0}^{r+1}(-)^{\alpha}\left(\prod_{\beta=1}^{\alpha} q_{\beta}^{\alpha-\beta}\right) \chi_{\alpha}\left(-z \frac{d}{d z}\right) z^{-\alpha} \psi(z)=0
$$

It is not difficult to find the coefficient in front of the highest derivative $d^{n} / d z^{n}$ in (2.18). Using (2.11) one can show that this coefficient has a nice factorized form

$$
\sum_{\alpha=0}^{r+1}(-)^{n+\alpha}\left(\prod_{\beta=1}^{\alpha} q_{\beta}^{\alpha-\beta}\right) \chi_{\alpha, 0} z^{n-\alpha}=(-)^{n} z^{n-r-1} \prod_{\alpha=0}^{r}\left(z-\prod_{\beta=1}^{\alpha} q_{\beta}\right)
$$

Further investigation confirms that indeed (2.18) is an $n$-th order Fuchsian differential equation with $r+3$ regular singular points located at

$$
z_{0}=\infty, z_{1}=1, z_{2}=q_{1}, z_{3}=q_{1} q_{2}, \ldots, z_{r+1}=q_{1} q_{2} \cdots q_{r}, z_{r+2}=0
$$

where for later use we have introduced new parameters $z_{\alpha}$ related to the gauge couplings through conditions

$$
q_{\alpha}=\frac{z_{\alpha+1}}{z_{\alpha}}
$$

\section{$2.1 \quad$ Exponents}

\subsubsection{Points $z_{r+2}=0$ and $z_{0}=\infty$}

First let's look after a solution of the form

$$
\psi(z)=z^{s}(1+O(z))
$$

Inserting this in (2.18) we see that when $z \rightarrow 0$ the term with $\alpha=r+1$ of (2.18) is the most singular one. So, for yet unknown constant $s$ we get the characteristic equation (sometimes called indicial equation)

$$
y_{r+1}(r+1-s)=0
$$

Similarly the characteristic equation for the infinity reads

$$
y_{0}(-s)=0
$$

2.1.2 Points $z_{1}=1, z_{2}=q_{1}, z_{3}=q_{1} q_{2}, \ldots, z_{r+1}=q_{1} q_{2} \cdots q_{r}$

Investigation of these points is slightly more subtle. Consider the ansatz

$$
\left.\psi(z)=\left(z-z_{\alpha}\right)^{s}\left(1+O\left(z-z_{\alpha}\right)\right)\right)
$$


for some fixed $\alpha \in\{1, \ldots, r+1\}$. Taking into account (2.19) and (2.12) for the index $s$ we get the equation

$$
\begin{aligned}
0= & (-)^{n} z_{\alpha}^{n-r-1} \prod_{\beta \neq \alpha, \beta=1}^{r+1}\left(z_{\alpha}-z_{\beta}\right) \prod_{i=0}^{n-1}(s-i) \\
& +\left(\sum_{\beta=0}^{r+1} n \beta\left(-z_{\alpha}\right)^{n-\beta-1} \sum_{1 \leq k_{1}<\cdots<k_{\beta} \leq r+1} \prod_{\gamma=1}^{\beta} z_{k_{\gamma}}\right) \prod_{i=0}^{n-2}(s-i) \\
& +\left(\sum_{\beta=0}^{r+1}\left(-z_{\alpha}\right)^{n-\beta-1} \sum_{1 \leq k_{1}<\cdots<k_{\beta} \leq r+1}\left(c_{0,1}-\sum_{\gamma=1}^{\beta}\left(c_{k_{\gamma-1,1}}-c_{k_{\gamma}, 1}\right)\right) \prod_{\gamma=1}^{\beta} z_{k_{\gamma}}\right) \prod_{i=0}^{n-2}(s-i) .
\end{aligned}
$$

The first two lines come from the terms proportional to $(z d / d z)^{n}$. The first (second) line includes part with $n$ "hits" $(n-1$ hits) on $\psi(z)$ by the operator $d / d z$. The third line is coming from the terms $\sim(z d / d z)^{n-1}$ with all $n$ operators $d / d z$ hitting $\psi(z)$. Though the second and especially third lines of this equation look quite complicated, fortunately they can be simplified drastically. Indeed it can be shown that the second line is equal to

$$
(-)^{n} n z_{\alpha}^{n-r-1} \prod_{\beta \neq \alpha, \beta=1}^{r+1}\left(z_{\alpha}-z_{\beta}\right) \prod_{i=0}^{n-2}(s-i)
$$

while the third line can be rewritten as

$$
(-)^{n} z_{\alpha}^{n-r-1}\left(c_{\alpha-1,1}-c_{\alpha, 1}\right) \prod_{\beta \neq \alpha, \beta=1}^{r+1}\left(z_{\alpha}-z_{\beta}\right) \prod_{i=0}^{n-2}(s-i) .
$$

Thus for the allowed exponents we get

$$
s \in\left\{0,1, \ldots n-2, c_{\alpha, 1}-c_{\alpha-1,1}-1\right\} .
$$

It is known in general that if indices differ by integers one might have logarithmic solutions. This is not the case however in the example at hand. A closer look ensures that our differential equation around $z=z_{\alpha}, \alpha=1,2, \ldots, r$ admits $n$ independent solutions of the types

$$
\begin{aligned}
& \left(z-z_{\alpha}\right)^{m}+O\left(\left(z-z_{\alpha}\right)^{n-1}\right) ; \quad m \in\{0,1,2, \ldots, n-2\} \quad \text { and } \\
& \left(z-z_{\alpha}\right)^{c_{\alpha, 1}-c_{\alpha-1,1}-1}\left(1+O\left(z-z_{\alpha}\right)\right) .
\end{aligned}
$$

\section{Toda CFT}

\subsection{Preliminaries on $A_{n-1}$ Toda CFT}

These are 2d CFT theories which, besides the spin 2 holomorphic energy momentum current $W^{(2)}(z) \equiv T(z)$ are endowed with additional higher spin $s=3 \ldots, n$ currents $W^{(3)}$, $\ldots W^{(n)}$ [36-38]. The Virasoro central charge is conventionally parameterised as

$$
c=n-1+12(Q, Q),
$$


where the "background charge" $Q$ is given by

$$
Q=\rho(b+1 / b),
$$

where $\rho$ is the Weyl vector of the algebra $A_{n-1}$ and $b$ is the dimensionless coupling constant of Toda theory. In what follows it would be convenient to represent roots, weights and Cartan elements of $A_{n-1}$ as $n$-component vectors subject to condition that sum of components is zero and endowed with the usual Kronecker scalar product. Obviously this is equivalent to a more conventional representation of these quantities as diagonal traceless $n \times n$ matrices with pairing given by trace. In this representation the Weyl vector is given by

$$
\rho=\left(\frac{n-1}{2}, \frac{n-3}{2}, \ldots, \frac{1-n}{2}\right)
$$

and for the central charge we'll get

$$
c=(n-1)\left(1+n(n+1) q^{2}\right)
$$

where for the later use we have introduced the parameter

$$
q=b+\frac{1}{b} .
$$

For further reference let us quote here explicit expressions for the highest weight $\omega_{1}$ of the first fundamental representation and for its complete set of weights $h_{1}, \ldots, h_{n}\left(h_{1}=\omega_{1}\right)$

$$
\begin{aligned}
& \left(\omega_{1}\right)_{k}=\delta_{1, k}-1 / n, \\
& \left(h_{l}\right)_{k}=\delta_{l, k}-1 / n .
\end{aligned}
$$

The primary fields $V_{\alpha}$ (in this paper we concentrate only on the left moving holomorphic parts) are parameterized by vectors $\alpha$ with vanishing center of mass. Their conformal wights are given by

$$
h_{\alpha}=\frac{(\alpha, 2 Q-\alpha)}{2} .
$$

In what follows a special role is played by the fields $V_{\lambda \omega_{1}}$ with dimensions

$$
h_{\lambda \omega_{1}}=\frac{\lambda(n-1)}{2}\left(q-\frac{\lambda}{n}\right) .
$$

For generic $\lambda$ these fields admit a single null vector at the first level.

\subsection{Fusion with the completely degenerated field $V_{-b \omega_{1}}$}

The field $V_{-b \omega_{1}}$ plays a special role in Toda theory. Fusion rules with this field are especially simple (see e.g. [39])

$$
V_{-b \omega_{1}}(z) V_{\alpha}(0)=\sum_{k=1}^{n} z^{b\left(Q_{1}-Q_{k}+\alpha_{k}\right)}\left[V_{\alpha-b h_{k}}(0)\right],
$$


where $h_{k}$ are the weights of the first fundamental representation and $\left[V_{\alpha}\right]$ denotes the $W$ class of the primary field $V_{\alpha}$. In the case when $V_{\alpha}$ is partially degenerated (i.e. $\alpha=\lambda \omega_{1}$ for some scalar $\lambda$ ), then in (3.4) only the first two terms contribute, all other classes drop out due to vanishing of the relevant structure constants. The remaining exponents are equal to respectively

$$
\frac{\lambda b(n-1)}{n} \quad \text { and } \quad b\left(q-\frac{\lambda}{n}\right) .
$$

In classical limit $(q \sim 1 / b)$ the field $V_{-b \omega_{1}}$ satisfies the $n$-th order differential equation $[39,40]$

$$
\sum_{k=0}^{n} w^{(n-k)}(z) \partial_{z}^{k} V_{-b \omega_{1}}=0,
$$

where $w^{(0)} \equiv 1, w^{(1)} \equiv 0$ and the other coefficients $w^{(n-k)}(z)$ are the expectation values of the currents $b^{n-k} W^{(n-k)}(z)$ in the classical limit $b \rightarrow 0$ (the prefactor $b^{n-k}$ is included to secure a finite limit).

\subsection{Derivation of null-vector decoupling equation in semiclassical limit}

Let us consider the semi-classical limit of the correlator

$$
\left\langle V_{\alpha^{(0)}}(\infty) V_{-b \omega_{1}}(z) V_{\alpha^{(1)}}\left(z_{1}\right) \cdots V_{\alpha^{(r+1)}}\left(z_{r+1}\right) V_{\alpha^{(r+2)}}(0)\right\rangle
$$

where we'll assume that all the fields besides $V_{-b \omega_{1}}(z)$ are "heavy", namely

$$
\alpha^{(0)}=\eta^{(0)} / b ; \quad \alpha^{(r+2)}=\eta^{(r+2)} / b ; \quad \alpha^{(k)}=\eta^{(k)} \omega_{1} / b
$$

(the parameters $\eta^{(0)}$ and $\eta^{(r+2)}$ are $n$-component vectors while $\eta^{(k)}$ are scalars all of them remaining finite in the $b \rightarrow 0$ limit). We have chosen all the parameters, besides the first and the last ones, to be proportional to the first fundamental weight $\omega_{1}$ since this is the case when the AGT relation between correlation functions and the partition function of quiver gauge theory holds. As we'll see later, the AGT correspondence in the $b \rightarrow 0$ limit emerges as a special case of a more general construction presented in the remaining part of this paper. In semiclassical limit the correlator (3.7) factorizes into a product of the classical (normalized) expectation value of the light field $V_{-b \omega_{1}}(z)$ with the correlator of the remaining heavy operators, hence, with respect to the variable $z$ it must satisfy the same differential equation (3.6):

$$
\left(\frac{d^{n}}{d z^{n}}+\sum_{k=2}^{n} w^{(k)}(z) \frac{d^{n-k}}{d z^{n-k}}\right) G(z)=0 .
$$

As already mentioned the coefficient functions $w^{(n-k)}(z)$ are the classical expectation values of the holomorphic currents $b^{n-k} W^{(n-k)}(z)$ in the background of heavy operators. Due to the form of OPE of $W^{(k)}$-current with primary fields, this classical expectation values should be rational functions of $z$, with $k$-th order poles located at the insertion points of 
heavy primary fields. The OPE (3.4) completely fixes the indices at the singular points as follows:

$$
\begin{array}{ll}
z=0 ; & u-1+\left(\eta^{(r+2)}\right)_{u} \text { for } u=1,2, \ldots, n \\
z=z_{\alpha} ; & 1-\eta^{(\alpha)} / n \text { and }(n-1) \eta^{(\alpha)} / n \text { for } \alpha=1,2, \ldots, r+1 \\
z=\infty ; & u-1+\left(\eta^{(0)}\right)_{u} \text { for } u=1,2, \ldots, n .
\end{array}
$$

There is a small puzzle to be understood here. The indicial equation at $z=z_{\alpha}$ is a degree $n$ algebraic equation while on the second line of eq. (3.10) we quoted only two indices. Multiple roots are not admissible, both field appearing on the r.h.s. of the OPE should come with multiplicity one. To find the missing indices, let us slightly change the charge parameters of the field at the point $z_{\alpha}$. We'll immediately see that besides two indices close to those given on the second line of (3.10), there are $n-2$ additional indices, located close to the points

$$
2-\eta^{(\alpha)} / n, 3-\eta^{(\alpha)} / n, \ldots, n-1-\eta^{(\alpha)} / n .
$$

Thus it is natural to assume that besides $(n-1) \eta^{(\alpha)} / n$ we have sequence of $n-1$ indices

$$
1-\eta^{(\alpha)} / n, 2-\eta^{(\alpha)} / n, \ldots, n-1-\eta^{(\alpha)} / n .
$$

The extra indices we got are naturally attributed to the contribution of descendant fields. This is not the end of story yet. It is well known that if there are indices at a singular point which differ from each other by integers, generally speaking logarithmic solutions emerge, something, which is not acceptable in a standard CFT such as Toda theory. The condition that logarithmic solutions are actually absent, imposes further restrictions on the rational coefficient functions $w^{(k)}(z)$. We'll explicitly parameterize these rational functions as

$$
w^{(k)}(z)=\sum_{\alpha=1}^{r+2} \sum_{m=0}^{k-1} \frac{w_{-m}^{(k, \alpha)}}{\left(z-z_{\alpha}\right)^{k-m}} .
$$

Consider a solution of the differential equation (3.9) around $z=z_{\alpha}$ represented as a series

$$
G(z)=\sum_{l=0}^{\infty} b_{l}\left(z-z_{\alpha}\right)^{l+s_{\alpha}}
$$

Let us insert this expansion into (3.6) and read off the first $n-1$ constraints imposed by the differential equation. We immediately get the relations

$$
b_{m}\left(s_{\alpha}+m-n+1\right)_{n}+\sum_{k=2}^{n} \sum_{l=m-k+1}^{m} b_{l} w_{m-l}^{(k, \alpha)}\left(s_{\alpha}+l-n+k+1\right)_{n-k}=0
$$

valid for $m \in\{0,1, \ldots, n-2\}$, where

$$
(x)_{l}=x(x+1) \cdots(x+l-1)
$$

is the Pochhammer's symbol. According to what has been discussed above, the coefficients $w^{(n-k)}(z)$ of the differential equation should be chosen so that these constraints be satisfied for

$$
s_{\alpha}=1-\frac{\eta^{(\alpha)}}{n}
$$


and for arbitrary constants $b_{0}, \ldots, b_{n-2}$. In particular choosing $b_{l}=\delta_{l, m}$ we arrive at

$$
\left(s_{\alpha}+m-n+1\right)_{n}+\sum_{k=2}^{n} w_{0}^{(k, \alpha)}\left(s_{\alpha}+m-n+k+1\right)_{n-k}=0,
$$

satisfied for each $m=0,1, \ldots, n-2$. Solving this system of equations with respect to $w_{0}^{(k, \alpha)}$ we get

$$
w_{0}^{(k, \alpha)}=(-1)^{k+1}\left(\begin{array}{l}
n \\
k
\end{array}\right)(k-1)\left(s_{\alpha}-1\right)_{k},
$$

where the standard notation

$$
\left(\begin{array}{l}
n \\
k
\end{array}\right)=\frac{n !}{k !(n-k) !}
$$

for the binomial coefficient is used. It is rewarding to see that this formula with parameter $s_{\alpha}$ specified in (3.17) gives correct zero mode eigenvalues of W-currents on the field $V_{\omega_{1} \eta_{\alpha} / b}$ in semiclassical limit $b \rightarrow 0$. In particular for the (rescaled) conformal dimension (i.e. for $k=2$ ) we get

$$
w_{0}^{(2, \alpha)}=\frac{(n-1) \eta^{(\alpha)}\left(1-\eta^{(\alpha)} / n\right)}{2}
$$

in complete agreement with (3.3). I have checked that also the other eigenvalues agree with data available in the literature.

The remaining constraints that follow from eq. (3.15) can be represented as

$$
\sum_{k=m+1}^{n} w_{-m}^{(k, \alpha)}\left(s_{\alpha}-n+k+l\right)_{n-k}=0, \quad \text { for } \quad l=1,2, \ldots, n-m-1
$$

valid for each value of $m \in\{1,2, \ldots, n-2\}$. These equations allow one to express all quantities $w_{-m}^{(k, \alpha)}$ with $k \in\{2,3, \ldots, n\}$ and $m \in\{1,2, \ldots, k-1\}$ in terms of $w_{-m}^{(m+1, \alpha)}$. Here is the explicit expression:

$$
w_{-m}^{(k, \alpha)}=(-1)^{k-m+1}\left(\begin{array}{c}
n-m-1 \\
n-k
\end{array}\right)\left(s_{\alpha}\right)_{k-m-1} w_{-m}^{(m+1, \alpha)} .
$$

Thus we managed to express all the coefficients of higher order poles at $z=z_{\alpha}, \alpha=$ $1,2, \ldots, r+1$ (but not those corresponding to $z=0$ ) in terms of the residues $w_{-m}^{(m+1, \alpha)}$.

Recall now that there should be one more independent index at $z=z_{\alpha}$ (see middle line of (3.10) and (3.17)) equal to

$$
\tilde{s}_{\alpha}=(n-1) \eta^{(\alpha)} / n=(n-1)\left(1-s_{\alpha}\right) .
$$

The indicial equation to be satisfied is (cf. (3.15)):

$$
\left(\tilde{s}_{\alpha}-n+1\right)_{n}+\sum_{k=2}^{n} w_{0}^{(k, \alpha)}\left(\tilde{s}_{\alpha}-n+k+1\right)_{n-k}=0 .
$$

It can be checked that quite remarkably this equality under substitutions (3.19), (3.23) is indeed satisfied automatically. 
It remains to take into account constraints imposed by Ward identities. These identities are consequences of ${ }^{5}$

$$
\left\langle\alpha^{(0)}\right| W_{m}^{(k)}=(-1)^{k+1} w_{0}^{(k, 0)} \delta_{m, 0} ; \quad m \geq 0
$$

and the commutation relations

$$
\left[W_{m}^{(k)}, V_{\alpha}(z)\right]=\sum_{l=1}^{k} z^{k+m-l}\left(\begin{array}{c}
k+m-1 \\
l-1
\end{array}\right) W_{l-k}^{(k, z)} V_{\alpha}(z),
$$

where

$$
W_{l}^{(k, z)} V_{\alpha}(z)=\oint_{z}(\zeta-z)^{l+k-1} W^{(k)}(\zeta) V_{\alpha}(z) \frac{d \zeta}{2 \pi i}
$$

is a descendant field on the level $-l$ for $l<0$. If $l>0,(3.27)$ vanishes, while for $l=0$

$$
W_{0}^{(k, z)} V_{\alpha}(z)=w_{0}^{(k)} V_{\alpha}(z)
$$

$w_{0}^{(k)}$ being the zero mode eigenvalue corresponding to the field $V_{\alpha}$. For $m>0$ combining (3.25) and (3.26) we easily get

$$
w_{-m}^{(k, r+2)}=-\sum_{\alpha=1}^{r+1} \sum_{l=1}^{k-m} z_{\alpha}^{k-m-l}\left(\begin{array}{c}
k-m-1 \\
l-1
\end{array}\right) w_{l-k}^{(k, \alpha)}
$$

or, in view of $(3.22)$

$$
w_{-m}^{(k, r+2)}=\sum_{\alpha=1}^{r+1} \sum_{l=1}^{k-m}(-)^{l} z_{\alpha}^{k-m-l}\left(\begin{array}{c}
k-m-1 \\
l-1
\end{array}\right)\left(\begin{array}{c}
n+l-k-1 \\
l-1
\end{array}\right)\left(s_{\alpha}\right)_{l-1} w_{l-k}^{(k-l+1, \alpha)} .
$$

Similarly analysing $m=0$ case of (3.25) and (3.26) we get the relation

$$
\begin{gathered}
(-1)^{k+1} w_{0}^{(k, 0)}+\sum_{\alpha=1}^{r+2} w_{0}^{(k, \alpha)} \\
+\sum_{\alpha=1}^{r+1} \sum_{l=1}^{k-1}(-)^{l+1} z_{\alpha}^{k-l}\left(\begin{array}{c}
k-1 \\
l-1
\end{array}\right)\left(\begin{array}{c}
n+l-k-1 \\
l-1
\end{array}\right)\left(s_{\alpha}\right)_{l-1} w_{l-k}^{(k-l+1, \alpha)}=0 .
\end{gathered}
$$

These equations allow one to express all coefficients related to the point $z_{1}=1$ (i.e. $w_{1-k}^{(k, 1)}$ ) in terms of coefficients $w_{1-k}^{(k, \alpha)}$ with $\alpha=2,3, \ldots, r+1$. Indeed the system (3.31) with respect to the variables $w_{1-k}^{(k, 1)}$ has a Gaussian triangular form and can be solved. As a side remark note that this problem reduces to the inversion of the $(n-1) \times(n-1)$ lower triangular matrix

$$
M_{i, j}=\frac{(s)_{i-j}}{((i-j) !)^{2}},
$$

\footnotetext{
${ }^{5}$ The sign factor $(-1)^{k+1}$ reflects the fact that a two-point function of primaries is non-zero if the zero modes of even spin currents (e.g. dimensions) coincide while those of odd currents have opposite signs (see e.g. [40]).
} 
where $i, j \in\{1,2, \ldots, n-1\}$. It's possible to show that the inverse matrix can be represented as

$$
\left(M^{-1}\right)_{i, j}=\sum_{l=0}^{n-2} P_{l}(s) \delta_{i-j, l},
$$

where the polynomials $P_{l}(s)$ are conveniently given by means of a generating function

$$
\sum_{l=0}^{\infty} P_{l}(s) q^{l}=\frac{1}{{ }_{1} F_{1}(s, 1, q)}
$$

with

$$
{ }_{1} F_{1}(a, b, q)=\sum_{l=0}^{\infty} \frac{(a)_{l}}{(b)_{l} l !} q^{l} .
$$

For the later reference let us write down $w^{(2)}(z)$ (see eq. (3.13)) in terms of dimensions and the parameters $w_{-1}^{(2, \alpha)}, \alpha=2,3, \ldots, r+1$, explicitly

$$
\begin{aligned}
w^{(2)}(z)= & \frac{w_{0}^{(2, r+2)}}{z^{2}}+\sum_{\alpha=1}^{r+1} \frac{w_{0}^{(2, \alpha)}}{\left(z-z_{\alpha}\right)^{2}} \\
& +\frac{w_{0}^{(2,0)}-\sum_{\alpha=1}^{r+2} w_{0}^{(2, \alpha)}}{z\left(z-z_{1}\right)}+\sum_{\alpha=2}^{r+1} \frac{z_{\alpha}\left(z_{\alpha}-z_{1}\right) w_{-1}^{(2, \alpha)}}{z\left(z-z_{1}\right)\left(z-z_{\alpha}\right)} .
\end{aligned}
$$

To conclude we succeeded to express all the parameters of the differential equation (3.9) in terms of (see eqs. (3.19), (3.22), (3.30) and (3.31)):

- zero-mode eigenvalues of the $W$-currents corresponding to the initial state, insertion fields and the final state (i.e. $w_{0}^{(k, \alpha)}$ for $k \in\{2,3, \ldots, n\}, \alpha \in\{0,1, \ldots, r+2\}$ )

- the coefficients $w_{1-k}^{(k, \alpha)}$ for $k \in\{2,3, \ldots, n\}, \alpha \in\{2,3, \ldots, r+1\}$, which are residues of rational functions $w^{(k)}(z)$ at the points $z=z_{\alpha}$

The parameters of the second list will be referred as the accessory parameters, since these are direct generalizations of the accessory parameters of the Liouville theory (the particular $n=2$ case of Toda theory) [41]. To avoid confusion notice that we have in mind not the "real" monodromy problem of $[41]$, but a generalization of the complex $\operatorname{SL}(2, \mathbb{C})$ monodromy problem, discussed in $[42,43]$, to the $\operatorname{SL}(n, \mathbb{C})$ case.

It is essential to note that there are exactly $(n-1) \times r$ accessory parameters, as many as the number of parameters necessary to fix $r$ intermediate $W$-families of the conformal block. We will see soon, that the DSW "curve" is the appropriate tool to solve the related monodromy problem, namely, to find such set of accessory parameters, which corresponds to a given set of intermediate $W$-families. In particular concentrating on the accessory parameters $w_{-1}^{(2, \alpha)}$, we will easily reestablish the famous AGT relations (in the semiclassical limit). Consideration of the remaining accessory parameters lead to a generalization of AGT, relating conformal blocks including non-primary fields on 2d CFT side with gauge invariant expectation values on the $\mathcal{N}=2$ gauge theory side. Investigation of this new relations in general quantum Toda case (e.g. along the lines of [44, 45], seems to be quite an interesting task. 


\section{Comparison with the differential equation derived from DSW}

\subsection{From the gauge theory differential equation to the null-vector decoupling equation}

We'll argue here that the differential equation obtained from (2.18) by substitution

$$
\psi(z)=G(z) \prod_{\alpha=1}^{r+2}\left(z-z_{\alpha}\right)^{t_{\alpha}}
$$

with suitably chosen parameters $t_{\alpha}$ coincides with the differential equation (3.9) satisfied by Toda CFT semi-classical conformal block (3.7). The idea is to choose parameters $t_{\alpha}$ so that the term with derivative of order $n-1$ disappears. Straightforward calculations show that this task is achievable with the unique choice

$$
\begin{aligned}
t_{r+2} & =r+1+\frac{1-n}{2}-\frac{c_{r+1,1}}{n} ; \\
t_{\alpha} & =\frac{c_{\alpha, 1}-c_{\alpha-1,1}}{n}-1, \quad \alpha=1,2,3, \ldots, r+1 .
\end{aligned}
$$

The resulting differential equation for $G(z)$ can be represented as

$$
\left(\frac{d^{n}}{d z^{n}}+S_{2}(z) \frac{d^{n-2}}{d z^{n-2}}+\cdots+S_{n}(z)\right) G(z)=0,
$$

where, in particular,

$$
\begin{aligned}
S_{2}(z)= & \left(\frac{n\left(n^{2}-1\right)}{24}-\frac{n-1}{2 n} c_{r+1,1}^{2}+c_{r+1,2}\right) \frac{1}{z^{2}} \\
& +\sum_{\alpha=1}^{r+1}\left(\frac{(n-1)\left(c_{\alpha, 1}-c_{\alpha-1,1}\right)\left(c_{\alpha-1,1}-c_{\alpha, 1}+n\right)}{2 n\left(z-z_{\alpha}\right)^{2}}\right. \\
& \left.+\frac{c_{\alpha-1,2}-c_{\alpha, 2}+\left(c_{\alpha-1,1}-c_{\alpha, 1}\right)\left(-c_{\alpha, 1}+\frac{c_{r+1,1}}{n}+\alpha+\frac{n-3}{2}-r\right)}{z\left(z-z_{\alpha}\right)}\right) \\
& +\sum_{1 \leq \alpha<\beta \leq r+1} \frac{\left(c_{\alpha-1,1}-c_{\alpha, 1}\right)\left(c_{\beta-1,1}-c_{\beta, 1}+n\right)}{n\left(z-z_{\alpha}\right)\left(z-z_{\beta}\right)} .
\end{aligned}
$$

In general $S_{u}(z)$ for $u=2,3, \ldots, n$, are rational functions with poles of order $u$ located at $z \in\left\{z_{1}=1, z_{2}, \ldots, z_{r+1}, z_{r+2}=0\right\}$.

Note that the substitution (4.1) shifts all exponents described in section 2.1 in an obvious manner. Namely the exponents at $z_{\alpha}$ get shifted by $t_{\alpha}$ for $\alpha=1,2, \ldots, r+2$ and the exponent at $z_{0}=\infty$ by

$$
t_{r+2}+\cdots+t_{r+1}=\frac{1-n}{2}-\frac{c_{0,1}}{n}
$$

so that the resulting indices become

$$
\begin{aligned}
& z=0 ; \quad \frac{n-1}{2}-a_{r+1, u}+\frac{c_{r+1,1}}{n}, \quad u=1,2, \ldots, n \\
& z=z_{\alpha} ; \quad u-\frac{c_{\alpha, 1}-c_{\alpha-1,1}}{n} \text { and } \quad \frac{(n-1)\left(c_{\alpha, 1}-c_{\alpha-1,1}\right)}{n}, \quad u=1,2, \ldots, n-1 \\
& z=\infty ; \quad \frac{n-1}{2}-a_{0, u}+\frac{c_{r+1,1}}{n}, \quad u=1,2, \ldots, n .
\end{aligned}
$$


Remind that $a_{0, u}$ and $a_{r+1, u}$ are the roots of the polynomials $y_{0}(x)$ and $y_{r+1}(x)$ respectively (see eqs. (2.4)). In particular

$$
\sum_{u=1}^{n} a_{0, u}=c_{0,1} \quad \text { and } \quad \sum_{u=1}^{n} a_{r+1, u}=c_{r+1,1}
$$

Now we see that the simple identification

$$
\begin{aligned}
\frac{n+1}{2}-u-\left(\eta^{(r+2)}\right)_{u} & \equiv a_{r+1, u}-\frac{c_{r+1,1}}{n} ; \\
\eta^{(\alpha)} & \equiv c_{\alpha, 1}-c_{\alpha-1,1} \\
\frac{n+1}{2}-u-\left(\eta^{(0)}\right)_{u} & \equiv a_{0, u}-\frac{c_{0,1}}{n}
\end{aligned}
$$

provides a perfect matching of indices (4.5) with (3.10).

\subsection{Matching $S_{2}(z)$ with $w^{(2)}(z)$ : emergence of AGT}

Now let us compare $S_{2}(z)$ (eq. (4.4)) with $w^{(2)}(z)$ (eq. (3.36)). Clearly the coefficients at the double poles under the map (4.7) become identical (not a surprise, since we have already checked that indices coincide).

Comparison of residues of poles at $z=z_{\alpha}$ for $\alpha \in\{2, \ldots, r+1\}$ leads to identification

$$
\begin{aligned}
w_{-1}^{(2, \alpha)}= & \frac{c_{\alpha-1,2}-c_{\alpha, 2}+\left(c_{\alpha-1,1}-c_{\alpha, 1}\right)\left(-c_{\alpha, 1}+\frac{c_{r+1,1}}{n}+\alpha+\frac{n-3}{2}-r\right)}{z_{\alpha}} \\
& +\sum_{\beta=1}^{\alpha-1} \frac{\left(c_{\beta-1,1}-c_{\beta, 1}\right)\left(c_{\alpha-1,1}-c_{\alpha, 1}+n\right)}{n\left(z_{\alpha}-z_{\beta}\right)}+\sum_{\beta=\alpha+1}^{r+1} \frac{\left(c_{\alpha-1,1}-c_{\alpha, 1}\right)\left(c_{\beta-1,1}-c_{\beta, 1}+n\right)}{n\left(z_{\alpha}-z_{\beta}\right)}
\end{aligned}
$$

Matching the residue at the pole $z=z_{1} \equiv 1$ requires

$$
\begin{array}{r}
w_{0}^{(2,0)}-\sum_{\alpha=1}^{r+2} w_{0}^{(2, \alpha)}-\sum_{\alpha=2}^{r+1} z_{\alpha} w_{-1}^{(2, \alpha)}= \\
c_{0,2}-c_{1,2}+\left(c_{0,1}-c_{1,1}\right)\left(-c_{1,1}+\frac{c_{r+1,1}}{n}+\frac{n-1}{2}-r\right) \\
+\sum_{\alpha=2}^{r+1} \frac{\left(c_{0,1}-c_{1,1}\right)\left(c_{\alpha-1,1}-c_{\alpha, 1}+n\right)}{n\left(1-z_{\alpha}\right)}
\end{array}
$$

A careful calculation shows that upon replacement of dimensions $w_{0}^{(2, \alpha)}$ by their expressions in terms of parameters $\eta^{(\alpha)}$ specified in (4.7) and substitution (4.8) for $w_{-1}^{(2, \alpha)}$, the eq. (4.9) becomes an identity. To prove this statement we have used the useful identity (for our 
purpose one should take $\left.d_{\gamma}=c_{\gamma, 1}-c_{\gamma-1,1}\right)$

$$
\begin{aligned}
& \sum_{\alpha=2}^{r+1} z_{\alpha}\left(\sum_{\beta=1}^{\alpha-1} \frac{d_{\beta}\left(n-d_{\alpha}\right)}{n\left(z_{\alpha}-z_{\beta}\right)}+\sum_{\beta=\alpha+1}^{r+1} \frac{d_{\alpha}\left(n-d_{\beta}\right)}{n\left(z_{\alpha}-z_{\beta}\right)}\right) \\
= & \int_{\mathcal{C}} \sum_{1 \leq \beta<\gamma \leq r+1} \frac{z d_{\beta}\left(n-d_{\gamma}\right)}{\left(z-z_{\beta}\right)\left(z-z_{\gamma}\right)} \frac{d z}{2 \pi i} \\
= & \sum_{1 \leq \beta<\gamma \leq r+1} d_{\beta}\left(n-d_{\gamma}\right)-\sum_{\gamma=2}^{r+1} \frac{d_{1}\left(n-d_{\gamma}\right)}{z_{1}-z_{\gamma}}
\end{aligned}
$$

where the contour $\mathcal{C}$ encloses the points $z_{2}, \ldots, z_{r+1}$ but not $z_{1}$. To pass from the second line to the third, one should notice that the same integral alternatively can be computed as sum of residues at infinity and at $z_{1}$ taken with negative signs.

There is no need to compare residues at the remaining pole at $z=z_{r+2}=0$ since all residues sum to zero (both $w^{(2)}(z)$ and $S_{2}(z)$ vanish at large values of $z$ as $1 / z^{2}$ ).

Now let us look on identification (4.8) more closely. The gauge theory expectation value

$$
\left\langle\operatorname{tr} \phi_{\alpha}^{2}\right\rangle=c_{\alpha, 1}^{2}-2 c_{\alpha, 2}
$$

is related to the partition function through Matone relation [46] which is valid also in presence of a nontrivial $\Omega$-background [47]:

$$
\left\langle\operatorname{tr} \phi_{\alpha}^{2}\right\rangle=\sum_{u=1}^{n} a_{\alpha, u}^{2}-b^{2} q_{\alpha} \partial_{q_{\alpha}} \log Z_{\text {inst }}
$$

where $Z_{\text {inst }}$ is the instanton part of the partition function and $a_{\alpha, u}$ are the Coulomb branch parameters which are related to the parameters $c_{\alpha, 1}$ by

$$
\sum_{u=1}^{n} a_{\alpha, u}=c_{\alpha, 1}=\left\langle\operatorname{tr} \phi_{\alpha}\right\rangle
$$

Notice also that in (4.12) taking into account our convention $\epsilon_{2}=1$ we have replaced

$$
\epsilon_{1} \epsilon_{2}=\frac{\epsilon_{1}}{\epsilon_{2}}=b^{2}
$$

where the last equality, as we'll see soon, is necessary to match the gauge theory and Toda theory sides. It will be convenient to separate the "center of mass" of the quantities $a_{\alpha, u}$ introducing new parameters (these are parameters indicated in figure $1 \mathrm{~b}$ )

$$
P_{\alpha, u}=a_{\alpha, u}-\frac{c_{\alpha, 1}}{n}
$$

Let me emphasize that the quantities $a_{\alpha, u}$ are genuine parameters of our gauge theory and as such, they do not depend on the gauge couplings $q_{\alpha}$ (or, equivalently, on $z_{\alpha}$ ). Thus, on r.h.s. of eq. (4.8), besides their explicit appearance, the variables $z_{\alpha}$ are hidden only in the differences $c_{\alpha-1,2}-c_{\alpha, 2}$. Due to $(2.20)$ we have

$$
q_{\alpha} \partial_{q_{\alpha}}=\sum_{\beta=\alpha+1}^{r+1} z_{\beta} \partial_{z_{\beta}} .
$$


Hence, combining (4.11), (4.12), (4.13) and (4.15) we get

$$
\begin{aligned}
c_{\alpha-1,2}-c_{\alpha, 2}= & \frac{1}{2} \sum_{u=1}^{n}\left(P_{\alpha, u}^{2}-P_{\alpha-1, u}^{2}\right)+\frac{n-1}{2 n}\left(c_{\alpha-1,1}^{2}-c_{\alpha, 1}^{2}\right) \\
& +b^{2} z_{\alpha} \partial_{z_{\alpha}} \log Z_{\text {inst }} .
\end{aligned}
$$

Inserting this expression into (4.8) after few simple manipulations, for $\alpha \in\{2, \ldots, r+1\}$ we obtain

$$
\begin{aligned}
w_{-1}^{(2, \alpha)}= & b^{2} \partial_{z_{\alpha}} \log Z_{\text {inst }}+\left(h\left(P_{\alpha-1, u}\right)-h\left(P_{\alpha, u}\right)-w_{0}^{(2, \alpha)}\right) \partial_{z_{\alpha}} \log z_{\alpha} \\
& -\sum_{\beta=1}^{\alpha-1} \eta^{(\beta)}\left(1-\frac{\eta^{(\alpha)}}{n}\right) \partial_{z_{\alpha}} \log \left(1-\frac{z_{\alpha}}{z_{\beta}}\right)-\sum_{\beta=\alpha+1}^{r+1} \eta^{(\alpha)}\left(1-\frac{\eta^{(\beta)}}{n}\right) \partial_{z_{\alpha}} \log \left(1-\frac{z_{\beta}}{z_{\alpha}}\right),
\end{aligned}
$$

where

$$
h\left(P_{\alpha, u}\right)=\frac{n\left(n^{2}-1\right)}{24}-\frac{1}{2} \sum_{u=1}^{n} P_{\alpha, u}^{2}
$$

are the (rescaled) dimensions with Toda momenta $P_{\alpha, u}$ (Toda momenta are related to the charge parameters via $\eta=\rho-P$ ) and dimensions $w_{0}^{(2, \alpha)}$ are given in (3.20). Remind now that $w_{-1}^{(2, \alpha)}$ is related to the Virasoro generator $b^{-2} L_{-1}$ acting on the field $V_{\lambda_{\alpha}}\left(z_{\alpha}\right)$. Thus

$$
w_{-1}^{(2, \alpha)}=b^{2} \partial_{z_{\alpha}} \log F_{\mathrm{CFT}},
$$

where $F_{\mathrm{CFT}}$ is the $r+3$ point conformal block of Toda theory depicted in figure $1 \mathrm{~b}$. Comparing (4.18) with (4.20) it is not hard to recognize the celebrated AGT relation.

The same technique, in principle, can be applied to investigate the remaining accessory parameters $w_{1-k}^{(k, \alpha)}$ with $k \in\{3, \ldots, n\}$. The result would be an extension of the AGT correspondence to the case of conformal blocks including a descendant field on CFT side and higher power expectation values in gauge theory side. Unfortunately in general case the next steps along this line seem to be quite complicated, one soon encounters awkward expressions. Instead in the next section we will give few more details for the simpler case of four point functions (i.e. for $r=1$ ).

\section{3 $A_{n-1}$-Toda 4-point functions versus $\mathrm{SU}(n)$ gauge theory with $2 n$ fundamen- tal hypers}

In this case we have a single DSW curve equation

$$
\chi_{1}(x)=\frac{q_{1} y_{0}(x-1) y_{2}(x)}{y_{1}(x-1)}+y_{1}(x)
$$

The first three coefficients of the $n$ 'th order polynomial $\chi_{1}(x)$ can be read off from the general formulae (2.11)-(2.13). We'll need also the forth coefficient which in our case $r=1$ 
is easy to obtain. Without loss of generality we can $\operatorname{set}^{6} c_{1,1}=0$. As a result we get

$$
\begin{aligned}
\chi_{1,0}= & 1+q_{1} ; \quad \chi_{1,1}=q_{1}\left(c_{0,1}+c_{2,1}\right) ; \\
\chi_{1,2}= & c_{1,2}+q_{1}\left(c_{0,2}-c_{1,2}+c_{0,1}\left(c_{2,1}-1\right)+c_{2,2}\right) ; \\
\chi_{1,3}= & c_{1,3}-q_{1}\left(c_{0,3}+2 c_{1,2}+c_{1,3}+c_{0,2}\left(c_{2,1}-2\right)-c_{1,2} c_{2,1}\right. \\
& \left.+c_{0,1}\left(-c_{1,2}-c_{2,1}+c_{2,2}+1\right)+c_{2,3}\right) .
\end{aligned}
$$

Now starting from the eq. (2.18) and following the steps described in previous sections we'll try to recast the differential equation in the form (4.3). The coefficient function $S_{2}(z)$ of (4.3) we have already calculated for the general case, so we'll simply specify the expression (4.4) to the case $r=1$.

The data (4.22) are sufficient for calculation of the next coefficient function $S_{3}(z)$ of (4.3). The calculation is rather lengthy but quite straightforward. Here we'll not give all the details. Those interested readers who might have desire to recover the results presented below could benefit from the useful identity

$$
\left(z \frac{d}{d z}\right)^{n}=\sum_{l=1}^{n} d_{n, l} z^{l}\left(\frac{d}{d z}\right)^{l},
$$

where the expansion coefficients admit a nice representation as

$$
d_{l+k, l}=\sum_{i_{1}=1}^{l} \sum_{i_{2}=1}^{i_{1}} \cdots \sum_{i_{l}=1}^{i_{l-1}} i_{1} i_{2} \cdots i_{l} .
$$

In fact, to calculate $S_{3}(z)$ one needs only the first four coefficients which can be easily deduced from (4.24)

$$
\begin{aligned}
& d_{n, 1}=1 ; \\
& d_{n, 2}=\frac{n(n-1)}{2} ; \\
& d_{n, 3}=\frac{n(n-1)(n-2)(3 n-5)}{24} ; \\
& d_{n, 4}=\frac{n(n-1)(n-2)^{2}(n-3)^{2}}{48} .
\end{aligned}
$$

It is clear from discussions in previous sections that knowing the residue of the rational function $S_{3}(z)$ at $z=z_{2}$ (i.e. $w_{-2}^{(3,2)}$ ) is sufficient to fully recover the function itself. Here is the final result for this residue

$$
w_{-2}^{(3,2)}=\frac{A}{\left(z_{2}-1\right)^{2}}+\frac{B}{z_{2}^{2}}+\frac{C}{z_{2}\left(z_{2}-1\right)},
$$

\footnotetext{
${ }^{6}$ In fact a uniform shift of all parameters $a_{\alpha, u}$ indicated in figure 1a is immaterial and can be compensated by the shift of same amount of the parameter $x$ of DSW equations (2.6).
} 
where

$$
\begin{aligned}
A= & \frac{2-n}{n^{2}} c_{0,1}\left(c_{0,1}+c_{2,1}\right)\left(n-c_{2,1}\right) \\
B= & c_{1,3}-c_{2,3}+(n-2) \\
& \times\left(\frac{1-n}{6 n^{2}} c_{2,1}\left(12 c_{2,1}^{2}-9 n c_{2,1}+2 n^{2}\right)+c_{1,2}\left(1-\frac{2 c_{2,1}}{n}\right)-c_{2,2}\left(1-\frac{3 c_{2,1}}{n}\right)\right) \\
C= & 2\left(1-\frac{c_{2,1}}{n}\right)\left(\left(\frac{2}{n}-1\right) c_{2,1} c_{0,1}-c_{0,1}+c_{0,2}-c_{1,2}\right) \\
& +c_{0,1}\left(n-1+\frac{2}{n}\left(c_{1,2}-c_{2,2}\right)\right) .
\end{aligned}
$$

Recall now that the parameters $c_{0,1}, c_{0,2}\left(c_{2,1}, c_{2,2}\right)$ are related to the fundamental (antifundamental) hyper-multiplet masses $m_{u},\left(\bar{m}_{u}\right)$ as

$$
\begin{aligned}
c_{2,1} & =\sum_{u=1}^{n} m_{u} ; & c_{2,2} & =\sum_{1 \leq u<v \leq n} m_{u} m_{v} ; \\
c_{0,1} & =\sum_{u=1}^{n} \bar{m}_{u} ; & c_{0,2} & =\sum_{1 \leq u<v \leq n} \bar{m}_{u} \bar{m}_{v} .
\end{aligned}
$$

Due to eq. (2.9)

$$
c_{1,2}=-\frac{1}{2}\left\langle\operatorname{tr} \phi^{2}\right\rangle ; \quad c_{1,3}=\frac{1}{3}\left\langle\operatorname{tr} \phi^{3}\right\rangle .
$$

Thus (4.26), (4.27) go beyond the standard AGT correspondance explicitly relating the semiclassical four-point function including a descendant field $W_{-2}^{(3)} V\left(z_{2}\right)$ with the gauge theory expectation values $\left\langle\operatorname{tr} \phi^{2}\right\rangle$ and $\left\langle\operatorname{tr} \phi^{3}\right\rangle$. The latter quantities can be calculated either with direct instanton calculus or DSW curve methods thus leading to the evaluation of the Toda descendant including four point conformal block. ${ }^{7}$

Conclusion. As a final remark note that from the mathematical point of view we have shown that:

- Any $n$ 'th order Fuchsian differential equation with generic ordinary singular points at 0 and $\infty$, additional $r+1$ ordinary points at $z_{1}=1, z_{2}, \ldots, z_{r+1}$ each of them obeying a set of indices of the form $\left\{0,1, \ldots, n-2, \xi_{\alpha}\right\}$ ( $\xi_{\alpha}$ are arbitrary), such that the equation doesn't accept any logarithmic solution can be represented in the form (2.18), where $\chi_{\alpha}(x)$ are $n$ 'th order polynomials with highest coefficient given by $(2.11),(2.20)$.

- The monodromy problem of such differential equation is intimately related to the system of difference equations (2.6) in the sense that the eigenvalues of the monodromy matrices $M_{\gamma} \in \mathrm{SL}(2, \mathbb{C})$ are given in terms of the period integrals of the meromorphic differentials $x d \log \left(y_{\alpha}(x)\right)$, where $y_{\alpha}(x)$ are the solutions of difference equations (2.6).

\footnotetext{
${ }^{7}$ Notice that this conformal block can not be related to the respective block of primaries via Ward identities, so that a straightforward CFT calculation is not available.
} 


\section{Acknowledgments}

I thank F. Fucito, J. F. Morales and G. Sarkissian for many interesting discussions. I am grateful to S. Theisen for hospitality at the AEI Potsdam-Golm where the bulk of this work has been completed and for a discussion. A discussion with S. Fredenhagen is also gratefully acknowledged. This work was supported by the Armenian State Committee of Science in the framework of the research project 15T-1C308 and by the Volkswagen Foundation of Germany.

Open Access. This article is distributed under the terms of the Creative Commons Attribution License (CC-BY 4.0), which permits any use, distribution and reproduction in any medium, provided the original author(s) and source are credited.

\section{References}

[1] N. Seiberg and E. Witten, Monopoles, duality and chiral symmetry breaking in $N=2$ supersymmetric QCD, Nucl. Phys. B 431 (1994) 484 [hep-th/9408099] [INSPIRE].

[2] N. Seiberg and E. Witten, Electric-magnetic duality, monopole condensation and confinement in $N=2$ supersymmetric Yang-Mills theory, Nucl. Phys. B 426 (1994) 19 [Erratum ibid. B 430 (1994) 485] [hep-th/9407087] [InSPIRE].

[3] A. Gorsky, I. Krichever, A. Marshakov, A. Mironov and A. Morozov, Integrability and Seiberg-Witten exact solution, Phys. Lett. B 355 (1995) 466 [hep-th/9505035] [INSPIRE].

[4] E.J. Martinec and N.P. Warner, Integrable systems and supersymmetric gauge theory, Nucl. Phys. B 459 (1996) 97 [hep-th/9509161] [INSPIRE].

[5] N.A. Nekrasov, Seiberg-Witten prepotential from instanton counting, Adv. Theor. Math. Phys. 7 (2003) 831 [hep-th/0206161] [INSPIRE].

[6] R. Flume and R. Poghossian, An Algorithm for the microscopic evaluation of the coefficients of the Seiberg-Witten prepotential, Int. J. Mod. Phys. A 18 (2003) 2541 [hep-th/0208176] [INSPIRE].

[7] U. Bruzzo, F. Fucito, J.F. Morales and A. Tanzini, Multiinstanton calculus and equivariant cohomology, JHEP 05 (2003) 054 [hep-th/0211108] [INSPIRE].

[8] N. Nekrasov and A. Okounkov, Seiberg-Witten theory and random partitions, Prog. Math. 244 (2006) 525 [hep-th/0306238] [INSPIRE].

[9] A. Lossev, N. Nekrasov and S.L. Shatashvili, Testing Seiberg-Witten solution, in Strings, branes and dualities. Proceedings, NATO Advanced Study Institute, Cargese, France, May 26 - June 14, 1997 [hep-th/9801061] [INSPIRE].

[10] L.F. Alday, D. Gaiotto and Y. Tachikawa, Liouville Correlation Functions from Four-dimensional Gauge Theories, Lett. Math. Phys. 91 (2010) 167 [arXiv:0906.3219] [INSPIRE].

[11] N. Wyllard, $A_{N-1}$ conformal Toda field theory correlation functions from conformal $N=2$ $\mathrm{SU}(N)$ quiver gauge theories, JHEP 11 (2009) 002 [arXiv:0907.2189] [INSPIRE].

[12] V.A. Fateev and A.V. Litvinov, Integrable structure, W-symmetry and AGT relation, JHEP 01 (2012) 051 [arXiv: 1109.4042] [INSPIRE]. 
[13] N.A. Nekrasov and S.L. Shatashvili, Quantization of Integrable Systems and Four Dimensional Gauge Theories, in Proceedings, 16th International Congress on Mathematical Physics (ICMP09) (2009) [arXiv:0908.4052] [INSPIRE].

[14] A. Mironov and A. Morozov, Nekrasov Functions and Exact Bohr-Zommerfeld Integrals, JHEP 04 (2010) 040 [arXiv:0910.5670] [INSPIRE].

[15] A. Mironov and A. Morozov, Nekrasov Functions from Exact BS Periods: The Case of $\mathrm{SU}(N)$, J. Phys. A 43 (2010) 195401 [arXiv:0911.2396] [InSPIRE].

[16] K. Maruyoshi and M. Taki, Deformed Prepotential, Quantum Integrable System and Liouville Field Theory, Nucl. Phys. B 841 (2010) 388 [arXiv:1006.4505] [InSPIRE].

[17] A. Marshakov, A. Mironov and A. Morozov, On AGT Relations with Surface Operator Insertion and Stationary Limit of Beta-Ensembles, J. Geom. Phys. 61 (2011) 1203 [arXiv: 1011.4491] [INSPIRE].

[18] R. Poghossian, Deforming SW curve, JHEP 04 (2011) 033 [arXiv: 1006.4822] [InSPIRE].

[19] F. Fucito, J.F. Morales, D.R. Pacifici and R. Poghossian, Gauge theories on $\Omega$-backgrounds from non commutative Seiberg-Witten curves, JHEP 05 (2011) 098 [arXiv:1103.4495] [INSPIRE].

[20] F. Fucito, J.F. Morales and D.R. Pacifici, Deformed Seiberg-Witten Curves for ADE Quivers, JHEP 01 (2013) 091 [arXiv: 1210.3580] [INSPIRE].

[21] N. Nekrasov, V. Pestun and S. Shatashvili, Quantum geometry and quiver gauge theories, arXiv: 1312.6689 [INSPIRE].

[22] R. Baxter, Exactly Solved Models in Statistical Mechanics, Academic Press, London (1982).

[23] V.V. Bazhanov, S.L. Lukyanov and A.B. Zamolodchikov, Integrable structure of conformal field theory. 3. The Yang-Baxter relation, Commun. Math. Phys. 200 (1999) 297 [hep-th/9805008] [INSPIRE].

[24] M. Piatek, Classical conformal blocks from TBA for the elliptic Calogero-Moser system, JHEP 06 (2011) 050 [arXiv:1102.5403] [INSPIRE].

[25] K. Bulycheva, H.-Y. Chen, A. Gorsky and P. Koroteev, BPS States in Omega Background and Integrability, JHEP 10 (2012) 116 [arXiv:1207.0460] [INSPIRE].

[26] M. Piatek, Classical torus conformal block, $N=2^{*}$ twisted superpotential and the accessory parameter of Lamé equation, JHEP 03 (2014) 124 [arXiv: 1309.7672] [INSPIRE].

[27] S.K. Choi, C. Rim and H. Zhang, Irregular conformal block, spectral curve and flow equations, JHEP 03 (2016) 118 [arXiv:1510.09060] [INSPIRE].

[28] G. Bonelli, A. Tanzini and J. Zhao, Vertices, Vortices and Interacting Surface Operators, JHEP 06 (2012) 178 [arXiv:1102.0184] [INSPIRE].

[29] G. Bonelli, A. Tanzini and J. Zhao, The Liouville side of the Vortex, JHEP 09 (2011) 096 [arXiv: 1107.2787] [INSPIRE].

[30] F. Fucito, J.F. Morales, R. Poghossian and D. Ricci Pacifici, Exact results in $\mathcal{N}=2$ gauge theories, JHEP 10 (2013) 178 [arXiv:1307.6612] [INSPIRE].

[31] M. Bershtein and O. Foda, AGT, Burge pairs and minimal models, JHEP 06 (2014) 177 [arXiv: 1404.7075] [INSPIRE]. 
[32] S.K. Ashok, M. Billó, E. Dell'Aquila, M. Frau, R.R. John and A. Lerda, Non-perturbative studies of $N=2$ conformal quiver gauge theories, Fortsch. Phys. 63 (2015) 259 [arXiv: 1502.05581] [INSPIRE].

[33] N. Nekrasov and V. Pestun, Seiberg-Witten geometry of four dimensional $N=2$ quiver gauge theories, arXiv:1211.2240 [INSPIRE].

[34] F. Cachazo, M.R. Douglas, N. Seiberg and E. Witten, Chiral rings and anomalies in supersymmetric gauge theory, JHEP 12 (2002) 071 [hep-th/0211170] [INSPIRE].

[35] F. Fucito, J.F. Morales and R. Poghossian, Wilson loops and chiral correlators on squashed spheres, JHEP 11 (2015) 064 [arXiv:1507.05426] [INSPIRE].

[36] A.B. Zamolodchikov, Infinite Additional Symmetries in Two-Dimensional Conformal Quantum Field Theory, Theor. Math. Phys. 65 (1985) 1205 [Teor. Mat. Fiz. 65 (1985) 347] [INSPIRE].

[37] V.A. Fateev and S.L. Lukyanov, The Models of Two-Dimensional Conformal Quantum Field Theory with Z(n) Symmetry, Int. J. Mod. Phys. A 3 (1988) 507 [InSPIRE].

[38] A. Bilal and J.-L. Gervais, Systematic Approach to Conformal Systems with Extended Virasoro Symmetries, Phys. Lett. B 206 (1988) 412 [INSPIRE].

[39] V.A. Fateev and A.V. Litvinov, On differential equation on four-point correlation function in the Conformal Toda Field Theory, JETP Lett. 81 (2005) 594 [Pisma Zh. Eksp. Teor. Fiz. 81 (2005) 728] [hep-th/0505120] [INSPIRE].

[40] V.A. Fateev and A.V. Litvinov, Correlation functions in conformal Toda field theory. I., JHEP 11 (2007) 002 [arXiv:0709.3806] [InSPIRE].

[41] L. Takhtajan and P. Zograf, Action for the Liouville equation as a generating function for the accessory parameters and as a potential for the Weil-Petersson metric on the Teichmüller space, Funkts Anal. Prilozh. 19 (1985) 67.

[42] N. Nekrasov, A. Rosly and S. Shatashvili, Darboux coordinates, Yang-Yang functional and gauge theory, Nucl. Phys. Proc. Suppl. 216 (2011) 69 [arXiv:1103.3919] [InSPIRE].

[43] A. Litvinov, S. Lukyanov, N. Nekrasov and A. Zamolodchikov, Classical Conformal Blocks and Painleve VI, JHEP 07 (2014) 144 [arXiv: 1309.4700] [INSPIRE].

[44] J.-E. Bourgine, Y. Mastuo and H. Zhang, Holomorphic field realization of SHc and quantum geometry of quiver gauge theories, arXiv:1512.02492 [INSPIRE].

[45] N. Nekrasov, BPS/CFT correspondence: non-perturbative Dyson-Schwinger equations and $q q$-characters, arXiv: 1512.05388 [INSPIRE].

[46] M. Matone, Instantons and recursion relations in $N=2$ SUSY gauge theory, Phys. Lett. B 357 (1995) 342 [hep-th/9506102] [INSPIRE].

[47] R. Flume, F. Fucito, J.F. Morales and R. Poghossian, Matone's relation in the presence of gravitational couplings, JHEP 04 (2004) 008 [hep-th/0403057] [INSPIRE]. 